\title{
Investigation by Digital Image Correlation of Mixed Mode I and II Fracture Behavior of Metallic IASCB Specimens with Additive Manufactured Crack-Like Notch
}

\author{
Ivo Campione ${ }^{1}$, Tommaso Maria Brugo ${ }^{2}$, Giangiacomo Minak ${ }^{1}{ }^{\mathbb{D}}$, Jelena Janković Tomić ${ }^{3}$, \\ Nebojša Bogojević ${ }^{3}$ and Snežana Ćirić Kostić ${ }^{3, *}$ \\ 1 Department of Industrial Engineering (DIN), Via Fontanelle 40, 47121 Forlì, Italy; \\ ivo.campione@unibo.it (I.C.); giangiacomo.minak@unibo.it (G.M.) \\ 2 Department of Industrial Engineering (DIN), University of Bologna, 40136 Bologna, Italy; \\ tommasomaria.brugo@unibo.it \\ 3 Faculty of Mechanical and Civil Engineering in Kraljevo, University of Kragujevac, 36000 Kraljevo, Serbia; \\ tomic.j@mfkv.rs (J.J.T.); bogojevic.n@mfkv.rs (N.B.) \\ * Correspondence: cirickostic.s@mfkv.kg.ac.rs; Tel.: +381-0648938826
}

Received: 11 February 2020; Accepted: 17 March 2020; Published: 20 March 2020

check for updates

\begin{abstract}
This work investigates the fracture behavior of maraging steel specimens manufactured by the selective laser sintering (SLS) technology, in which a crack-like notch (sharp notch) was directly produced during the additive manufacturing (AM) process. For the evaluation of the fracture toughness, the inclined asymmetrical semi-circular specimen subjected to three points loading (IASCB) was used, allowing to cover a wide variety of Mode I and II combinations. The effectiveness of manufacturing crack-like notches via the SLS technique in metals was evaluated by comparing the obtained experimental results with the ones obtained with pre-cracks induced by fatigue loading. The investigation was carried out by using the digital image correlation (DIC) technique, that allowed the evaluation of the full displacement fields around the crack tip. The displacement field was then used to compute the stress intensity factors (SIFs) for various combinations of Mode I and II, via a fitting technique which relies on the Williams' model for the displacement. The SIFs obtained in this way were compared to the results obtained with the conventional critical load method. The results showed that the discrepancy between the two methods reduces by ranging from Mode I to Mode II loading condition. Finally, the experimental SIFs obtained by the two methods were described by the mixed mode local stress criterium.
\end{abstract}

Keywords: additive manufacturing; selective laser sintering; maraging steel; fracture toughness; stress intensity factors; mixed mode I-II; IASCB specimens; digital image correlation; Williams' model; local stress criterium

\section{Introduction}

Additive manufacturing (AM) is a powerful technique that allows one to manufacture complex geometries, which would be difficult or impossible to obtain with the traditional subtractive techniques. Among the AM techniques, selective laser sintering (SLS) is one of the most widespread. It exploits a laser beam to sinter powder to build objects bottom-up, layer-by-layer and has the advantage of being applicable to a large variety of materials [1] such as metals, polymers, ceramics or wax. However, one drawback of the SLS technique is that the mechanical properties of the product depend on the direction in which the geometry is built up, which is to be properly chosen, especially when 
designing structural components [2]. This work investigates the fracture mechanics properties of SLS metallic materials and the capability of the AM processes to build 3-D artificial crack-like notch into them. This less conventional method could be used to induce internal crack-like notches in the specimens and study three-dimensional fracture mechanics problems, which are difficult to be tackled by conventional methods. Previous study of T. Brugo et al. [3] on fracture toughness of AM Polyamide materials have shown that it is possible to intentionally induce crack-like notches in any desired direction during the AM process and that the building direction slightly influences the fracture toughness.

In literature, several configurations are proposed to investigate mixed mode I/II fracture behavior. Two main types of configurations can be found: the first one exploits the use of asymmetrical rectangular specimens, such as [4,5] or the modified compact tension (CT) specimen [6]. The second one involves the use of disc-like specimens, such as the Brazilian Disc (BD) [7], the Semi-Circular Bend (SCB) [8] specimen, the Asymmetric Semi-Circular Bend (ASCB) $[9,10]$ specimen and the Inclined edge cracked Semi-Circular Bend (IASCB) [11,12] specimen. In this work the IASCB specimen, subjected to three-point bend loading, was utilized (the test configuration is described in detail in $\S 2.2$ ). This specimen, compared to the other ones reported, gives the opportunity to cover a wider range of the stress intensity factors $\left(K_{I}\right.$ and $\left.K_{I I}\right)$ and T-Stress without requiring a complicated loading fixture.

IASCB specimens were manufactured by SLS with different crack-like notch angles and compared with others in which the crack was induced by fatigue loading, considering different values of support spans. To evaluate the fracture behavior, the digital image correlation technique (DIC) was exploited. Along with the electronic speckle pattern interferometry (ESPI) [13], the DIC is one of the most widespread optical technique that allows to easily and cheaply measure the full displacement and strain field [14]. It requires just a camera of sufficiently high resolution and an algorithm to perform the correlation between the images, which is carried out by exploiting a speckle pattern (usually spray-painted) on the surface of the sample. This pattern serves as an aid for the correlation algorithm, which relies on matching subsets of pixels in consecutive images: the location of a point in an undeformed subset is found in the deformed image, and the displacement can thus be determined.

By means of this technique, the displacement field is computed directly, whereas the strain field is calculated by numerical differentiation of the former, with inevitable introduction of noise. Mainly for this reason, fitting methods usually exploit the displacement field rather than the strain one. In fracture mechanics, these displacement fields, other than providing a complete qualitative insight on the material behavior in the zone of interest (namely the crack tip, in the case study) can be used to quantitatively characterize the fracture behavior of the material. In particular, they can be exploited to estimate the stress intensity factors (SIFs). One notable work in this direction was done by McNeill et al. [15] who tested CT and Three Point Bending (3PB) specimens, and compared the results between DIC and analytical data, suggesting a methodology to use the DIC data to determine the fracture toughness $K_{I C}$ of specimens. In general, two main approaches emerges in literature concerning the fitting of theoretical models on the full displacement fields: the first one is based on guessing a general form of an analytical function and fitting this to the displacement experimental data: an example is the complex function analysis of Muskhelishvili [16], which was further developed to calculate mixed mode (I + II) [17] and generalized to cases without restrictions in boundary conditions or symmetry [18]. The second approach is based on the Williams' model [19-22], where the fitting on the experimental data is done by considering the first $n$ term of the Williams expansion for the displacement. In this work this latter approach was used. The SIFs obtained by the DIC fitting were compared to the ones obtained with the conventional critical load method. Finally, the resulting SIFs were described by the mixed mode local stress criterium. 


\section{Materials and Methods}

\subsection{Specimen Fabrication by Additive Manufacturing}

The specimens were manufactured by the EOSINT M 280 SLS machine (EOS GmbH Electro Optical Systems, Krailling/Munich, Germany), using the EOS Maraging Steel MS1 steel powder, with a chemical composition corresponding to the European 1.2709 classification. The process parameters were set as described in [23] and as advised by the SLS machine manufacturer.

In particular, the layer thickness was set to $40 \mu \mathrm{m}$ and the scanning pattern of each layer was rotated around the vertical axis with respect to the previous one, in order to reduce in-plane anisotropy. After the laser sintering process, the specimens underwent a surface cleaning treatment by micro shot-peening, followed by an ageing treatment by a heating in air up to $490{ }^{\circ} \mathrm{C}$ for $6 \mathrm{~h}$, followed again by micro shot-peening. According to the powder supplier, after the age-hardening treatment, the material anisotropy can be completely removed, and the excellent mechanical properties described in Table 1 can be attained.

Table 1. Mechanical properties of the specimens.

\begin{tabular}{cc}
\hline Property & Value \\
\hline Sintered Density & $8.0-8.1 \mathrm{~g} / \mathrm{cm}^{3}$ \\
Elastic Modulus & $180 \pm 20 \mathrm{GPa}$ \\
Yield Strength & $\min .1862 \mathrm{MPa}$ \\
Tensile Strength & $\min .1930 \mathrm{MPa}$ \\
Hardness & typ. $50-56 \mathrm{HRC}$ \\
Ductility (Notched Charpy Impact Test) & $11 \pm 4 \mathrm{~J}$ \\
\hline
\end{tabular}

\subsection{IASB Specimens}

The IASCB specimen, shown in Figure 1 is a semi-circular disk of radius $R$ and thickness $t$ which contains a radial edge crack of length $a$, tilted with respect to the load direction of an angle $\alpha$ [11]. The specimens were manufactured by the SLS technique with the built plane parallel to the plane of the semi-circumference of the specimen.

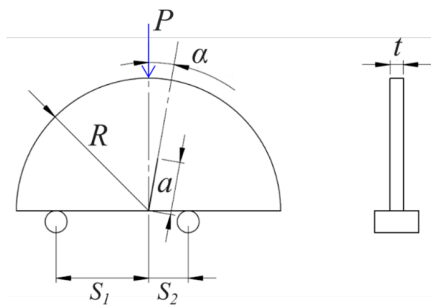

Figure 1. Geometrical parameters and loading conditions of the IASCB (Inclined edge cracked Semi-Circular Bend) specimen.

To conduct the fracture tests, the specimens were located on two bottom supports and loaded by a vertical force $P$. The existence of different geometric parameters allows various combinations of Mode I and Mode II to be easily achieved. The geometrical parameters which can be varied to achieve mixed mode (I and II) are:

(1) $S_{1} / R$ and $S_{2} / R$ (the ratio between the support spans and the specimen radius);

(2) $a / R$ (the ratio between the crack length and the specimen radius);

(3) $\alpha$ (the angle between the crack line and the load direction).

In order to obtain the various mode combinations, only the parameters $S_{2} / R$ and $\alpha$ were varied. $S_{1}$ was maintained fixed at $42 \mathrm{~mm}$, whereas the values of $t, R$ and $a$ were the same for all the specimens 
$(t=6 \mathrm{~mm}, R=60 \mathrm{~mm}, a=24 \mathrm{~mm})$ The various combinations of these two, indeed, can cover Mode I, Mode II and mixed modes I-II. When the bottom supports are located symmetrically to the crack line (i.e., when $S_{1}=S_{2}$ ) and the crack line is in the same direction as the load (i.e., when $\alpha=0^{\circ}$ ), the specimen is subjected to Mode I (opening mode). To obtain mixed mode I-II or Mode II (sliding mode), an appropriate combination of $S_{2} / R$ and $\alpha$ should be chosen.

The notch was initiated in two different ways: 16 samples had the crack-like notch manufactured during the additive manufacturing process (henceforth named AM specimens), while the remaining 3 samples had the notch initiated afterwards, by subtractive manufacturing (henceforth named SM specimens). In the SM specimens, the notch was induced by wire electrical discharge machining (EDM) technique; they were then fatigue pre-cracked (loading ratio $r=K_{\operatorname{Imin}} / K_{\operatorname{Imax}}=0.3$ ) until the crack propagated for $2 \mathrm{~mm}$. In Figure 2 micrographs of the notch induced with the two different methods are shown. The crack-like notch induced by the AM technique has a radius of about $90 \mu \mathrm{m}$, whereas the notch induced by SM has a radius of about $150 \mu \mathrm{m}$. The AM specimens were manufactured with an angle $\alpha$ of $0^{\circ}$ and $10^{\circ}$ and tested with different support spans $S_{1}$ and $S_{2}$ (see Figure 1), in order to obtain different mode combinations. The AM specimens with crack-like notch angle $\alpha$ of $0^{\circ}$ were only tested with symmetric support spans to pre-crack and then monotonic loaded in Mode I. Table 2 reports the specimens tested with the method employed to induce cracks, the values of $\alpha$, the supports spans and the mixed mode ratio expressed as $M^{e}=2 / \pi \operatorname{atan}\left(K_{I} / K_{I I}\right)$.
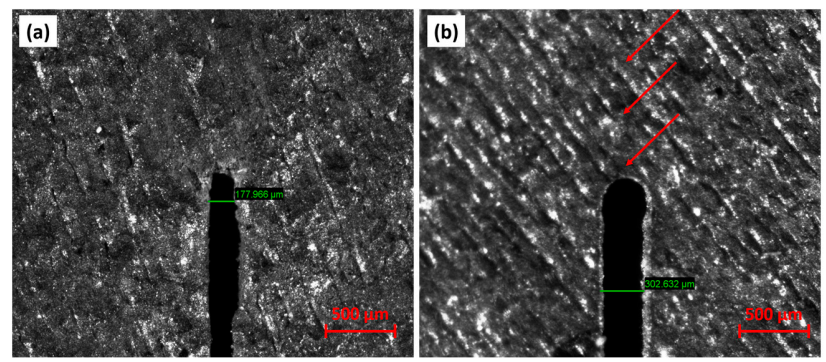

Figure 2. Micrographs of the crack tip; (a) crack-like notch manufactured during the additive manufacturing process (AM) and (b) crack induced by subtractive manufacturing (SM) and subsequent pre-cracking (indicated by red arrows).

Table 2. Values of the geometrical parameters of the specimens tested.

\begin{tabular}{ccccccc}
\hline Type & $\alpha \mathbf{( d e g})$ & $\boldsymbol{S}_{\mathbf{2}}(\mathbf{m m})$ & $\boldsymbol{S}_{\boldsymbol{1}}(\mathbf{m m})$ & $\mathbf{n}$ & Mode & $\boldsymbol{M}^{\boldsymbol{e}}$ \\
\hline SM & 0 & 42 & 42 & 3 & Mode I & 1 \\
AM & 0 & 42 & 42 & 3 & Mode I & 1 \\
AM & 10 & 42 & 42 & 3 & Mixed I-II & 0.77 \\
AM & 10 & 42 & 18 & 3 & Mixed I-II & 0.48 \\
AM & 10 & 42 & 10.2 & 3 & Mode II & 0.12 \\
\hline
\end{tabular}

\subsection{Experimental Setup}

The fracture tests were performed in displacement control at a speed of $1 \mathrm{~mm} / \mathrm{min}$, by using a hydraulic tensile machine Instron 8033 (Instron, Norwood, MA, USA) equipped with a $250 \mathrm{kN}$ load cell. The setup was arranged as shown in Figure 3. The 2D DIC system, used to measure the full displacement field around the crack tip (in a window of $14 \mathrm{~mm} \times 10 \mathrm{~mm}$ ), consisted in a $10 \mathrm{MP}$ Basler ace acA3800-14uc camera (Basler, Ahrensburg, Germany) equipped with Basler lens C125-2522-5M-P f25mm (Basler, Ahrensburg, Germany) and two custom 40W $6000 \mathrm{~K}$ led lamps. The images were acquired at $2 \mathrm{~Hz}$ with the GOM Snap 2D free software and processed by GOM Correlate (GOM GmbH, Braunschweig, Germany, https://www.gom.com/), with the correlation parameters set as follows: facet size of 19 pixel, point distance of 16 pixel, and spatial and temporary filter (median) set to 8 and 3 respectively. The correlation parameters were chosen based on the experimental DIC optimization work of Palanca et al. [24] as the best compromise between precision and spatial resolution, to evaluate 
the displacement field near the crack tip. In order to make the digital image correlation possible, a black speckle pattern with a white background was spray-painted on the specimens. Figure $3 b, c$ show an example of the correlated displacement and strain field normal to the crack axis, correlated by the DIC software.

(a)

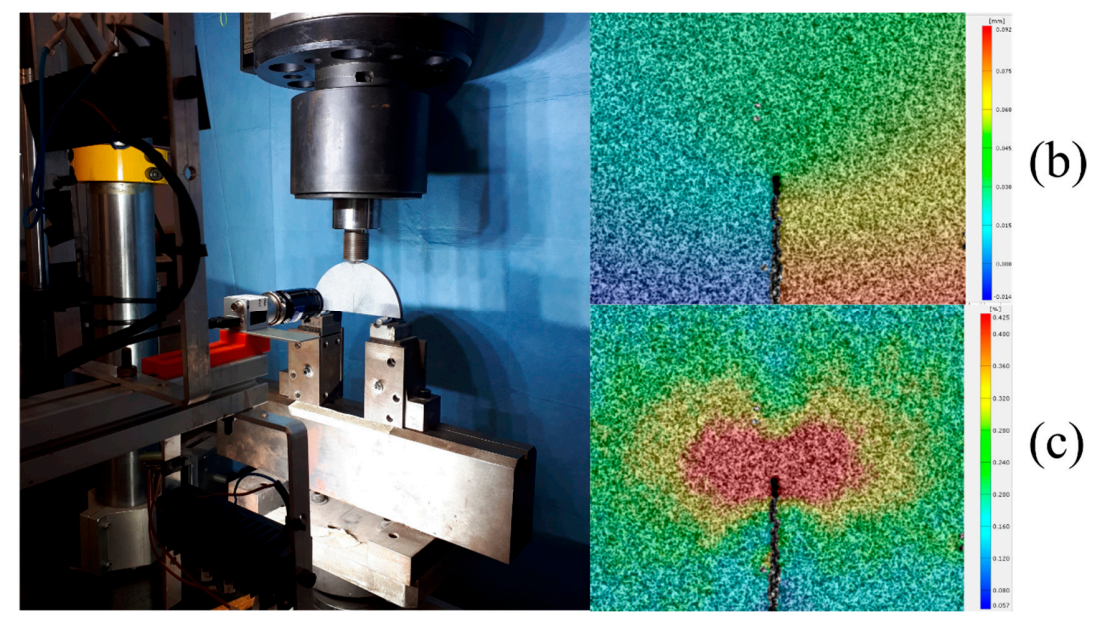

Figure 3. (a) Experimental setup; (b) crack axis normal displacement and (c) strain field correlated by the GOM software around the crack tip, for Mode I.

\section{Results}

\subsection{Evaluation of Stress Intensity Factors by the Critical Fracture Load Method}

For the IASCB specimens, the stress intensity factor in mixed modes I-II were determined by exploiting the finite element method presented [10]. According to this method, the loading conditions can be written as shown in Equations (1) and (2).

$$
\begin{aligned}
K_{I} & =\frac{P}{2 R t} \sqrt{\pi a Y_{I}}(a / R, S 1 / R, S 2 / R, \alpha) \\
K_{I I} & =\frac{P}{2 R t} \sqrt{\pi a Y_{I I}}(a / R, S 1 / R, S 2 / R, \alpha)
\end{aligned}
$$

where $Y_{\mathrm{I}}$ and $Y_{\mathrm{II}}$ are geometry factors corresponding to Mode I and Mode II, respectively (reported in Table 2 of reference [10]). Concerning the crack length $a$, for the SM specimens it was measured from the bottom of the sharp notch to the pre-crack tip, whereas for the AM specimens it was considered as the length of the crack-like notch. For each test, the critical fracture load $\left(P_{\mathrm{cr}}\right)$ from the load-displacement curves was evaluated at the instant in which the crack propagation was visually observed, by the camera pointing at the crack tip. Then, by Equations (1) and (2) the critical stress intensity factors $\left(K_{\mathrm{I}}\right.$ and $\left.K_{\mathrm{II}}\right)$ of the tested IASCB specimens were calculated. The obtained fracture parameters from the critical fracture load method (henceforth named PCR) are reported in Table 3. 
Table 3. Values of the SIFs obtained by critical fracture load and by fitting the displacement field.

\begin{tabular}{|c|c|c|c|c|c|c|}
\hline \multirow{2}{*}{$\begin{array}{l}\text { Specimen } \\
\text { Configuration }\end{array}$} & \multicolumn{2}{|c|}{ By Critical Fracture Load (PCR) } & \multicolumn{2}{|c|}{$\begin{array}{c}\text { By Crack Tip Displacement } \\
\text { Field (DIC) }\end{array}$} & \multicolumn{2}{|c|}{ DIC vs. PCR } \\
\hline & $K_{I}(\mathrm{MPa} \sqrt{\mathrm{m}})$ & $K_{\mathrm{II}}(\mathrm{MPa} \sqrt{\mathrm{m}})$ & $K_{I}(\mathrm{MPa} \sqrt{\mathrm{m}})$ & $K_{I I}(\mathrm{MPa} \sqrt{\mathrm{m}})$ & $\Delta K_{I}(\%)$ & $\Delta K_{I I}(\%)$ \\
\hline SM-0-42-42-1 & 32.1 & 0.0 & 58.3 & 1.2 & 81 & - \\
\hline SM-0-42-42-2 & 30.4 & 0.0 & 56.1 & 2.9 & 85 & - \\
\hline SM-0-42-42-3 & 33.7 & 0.0 & 60.5 & 2.3 & 80 & - \\
\hline AM-0-42-42-1 & 42.5 & 0.0 & 73.5 & 0.2 & 73 & - \\
\hline AM-0-42-42-2 & 44.9 & 0.0 & 76.4 & 0.1 & 70 & - \\
\hline AM-0-42-42-3 & 46.6 & 0.0 & 75.2 & 0.2 & 61 & - \\
\hline AM-10-42-42-1 & 44.4 & 16.5 & 70.5 & 20.5 & 59 & 25 \\
\hline AM-10-42-42-2 & 44.6 & 16.5 & 66.2 & 18.3 & 49 & 11 \\
\hline AM-10-42-42-3 & 45.1 & 16.7 & 70.2 & 18.3 & 56 & 10 \\
\hline AM-10-42-18-1 & 33.7 & 36.1 & 43.7 & 42.2 & 30 & 17 \\
\hline AM-10-42-18-2 & 36.3 & 38.8 & 39.2 & 43.4 & 8 & 12 \\
\hline AM-10-42-18-3 & 34.4 & 36.9 & 44.2 & 45.7 & 28 & 24 \\
\hline AM-10-42-10.2-1 & 15.3 & 83.5 & 14.9 & 77.1 & -2 & -8 \\
\hline AM-10-42-10.2-2 & 13.6 & 74.2 & 14.4 & 75.5 & 5 & 2 \\
\hline AM-10-42-10.2-3 & 14.2 & 79.4 & 14.9 & 80.8 & 5 & 2 \\
\hline
\end{tabular}

\subsection{Evaluation of Stress Intensity Factors by the DIC Full Displacement Field}

To model the displacement near the crack tip, the Williams' asymptotic formulation [25] was exploited. The $u$ and $v$ are the displacement along the crack axis (x-axis) and along an axis perpendicular to the former and contained in the crack plane (y-axis). Figure 4 show the $u$ and $v$ displacement field measured by the DIC and postprocessed in MATLAB ${ }^{\circledR}$ R2019b (MathWorks Inc., Natick, MA, USA) along with the frame of reference used, with the origin located into the crack tip and the axis oriented as shown in Figure 4c.

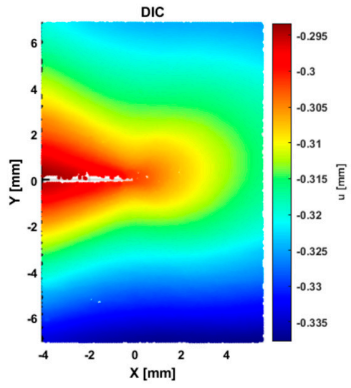

(a)

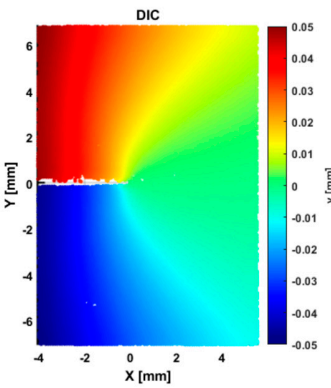

(b)

Figure 4. (a) x-displacement $(u)$ and (b) y-displacement $(v)$ of the AM-0-42-42-1 specimen obtained by the DIC (digital image correlation). (c) Position and orientation of the frame of reference used.

For Mode I, $u$ and $v$ are computed as functions of the polar coordinates $(r, \theta)$, according to the infinite series of Equations (3) and (4).

$$
\begin{aligned}
& u_{I}=\sum_{n=1}^{\infty} \frac{r^{\frac{n}{2}}}{2 \mu} a_{n}\left\{\left[\kappa+\frac{n}{2}+(-1)^{n}\right] \cos \frac{n \theta}{2}-\frac{n}{2} \cos \frac{(n-4) \theta}{2}\right\} \\
& v_{I}=\sum_{n=1}^{\infty} \frac{r^{\frac{n}{2}}}{2 \mu} a_{n}\left\{\left[\kappa-\frac{n}{2}-(-1)^{n}\right] \sin \frac{n \theta}{2}+\frac{n}{2} \sin \frac{(n-4) \theta}{2}\right\}
\end{aligned}
$$

For Mode II, $u$ and $v$ are computed as functions of the polar coordinates $(r, \theta)$, according to the infinite series of Equations (5) and (6). 


$$
\begin{aligned}
& u_{I I}=-\sum_{n=1}^{\infty} \frac{r^{\frac{n}{2}}}{2 \mu} b_{n}\left\{\left[\kappa+\frac{n}{2}-(-1)^{n}\right] \sin \frac{n \theta}{2}-\frac{n}{2} \cos \frac{(n-4) \theta}{2}\right\} \\
& v_{I I}=\sum_{n=1}^{\infty} \frac{r^{\frac{n}{2}}}{2 \mu} b_{n}\left\{\left[\kappa-\frac{n}{2}+(-1)^{n}\right] \cos \frac{n \theta}{2}+\frac{n}{2} \cos \frac{(n-4) \theta}{2}\right\}
\end{aligned}
$$

where $\mu=E /(2+2 v)$ is the shear modulus and $\kappa=(3-v) /(1+v)$ for plane stress and $\kappa=3-4 v$ for plane strain condition; $a_{\mathrm{n}}$ and $b_{\mathrm{n}}$ are the model parameters. In this analysis, the specimen was considered subjected to plane stress condition, which is an assumption justified by the specimen geometry and loading conditions [20].

For mixed modes, the displacement fields can be computed as the superimposition of the displacement of Mode I and Mode II, as shown in Equations (7) and (8).

$$
\begin{aligned}
& u=u_{I}+u_{I I} \\
& v=v_{I}+v_{I I}
\end{aligned}
$$

The values of KI and KII are related to the value of the Williams model parameters as shown in Equations (9) and (10).

$$
\begin{gathered}
K_{I}=a_{1} \sqrt{2 \pi} \\
K_{I I}=-b_{1} \sqrt{2 \pi}
\end{gathered}
$$

To perform the fitting, not only the parameters $a=a_{1}, \ldots, a_{\mathrm{n}}$ and $b=b_{1}, \ldots, b_{\mathrm{n}}$ were considered, but also four additional parameters were accounted to compensate rigids motions, which is an approach used also in $[19,20]$. In particular, the three translations and the rotation around the z-axis (perpendicular to the crack plane) were considered. As explained in [19], this approach allows one to tackle situations in which the material exhibits small-scale plastic deformation, but makes the fitting problem non-linear in the fitting parameters, because of the $x$ and $y$ translations $x_{0}$ and $y_{0}$, are embedded in the calculus of the polar coordinates $(r, \theta)$ as shown in Equations (11) and (12). This approach was particularly useful in the case of the SM specimens, because the fact that they were pre-cracked would have made difficult a precise manual identification of the crack tip.

$$
\begin{gathered}
r=\sqrt{\left(x-x_{0}\right)^{2}+\left(y-y_{0}\right)^{2}} \\
\theta=\operatorname{arctg}\left(\frac{y-y_{0}}{x-x_{0}}\right)
\end{gathered}
$$

The fitting was performed by a code developed in MATLAB ${ }^{\circledR}$ by the authors, which exploits the function "lsqcurvefit". The code is made publicly available at the link provided in the "Supplementary Materials" section. To perform the fitting, it was decided to utilize the y-displacement $v$, since is the one generally used to determinate the SIFs for Mode I, and for mixed mode problems the dominant displacement component for the crack cannot be known in advance [19].

A plasticization radius of 0.51 and $0.83 \mathrm{~mm}$ was calculated for Mode I and II respectively, according to the following equations: $r_{p}=1 / \pi\left(K_{I} / \sigma_{Y S}\right)^{2}$ and $r_{P I I}=3 /(2 \pi)\left(K_{I I} / \sigma_{Y S}\right)^{2}$ where $\sigma_{Y S}$ is the yield strength of the material reported in Table 1 and $K_{I}$ and $K_{I I}$ are the SIFs determined by the DIC method reported in Table 3 (considering the AM specimens in the case of Mode I and Mode II). However, we decided to only exclude the DIC data within $0.20 \mathrm{~mm}$ from the crack face (for every mode mixity), because they were very noisy and not reliable there, but to keep all the other data in the fitting. Indeed, the employed fitting method, in which the crack tip position is treated as an unknow quantity, can effectively provide the SIFs for small-scale yielding, as it was shown by Yoneyama et al. [19]. These assumptions are furthermore justified by the fact that the specimen material exhibits a mainly fragile behavior, as reported in [26]. 
Figure 5 shows the SIFs values obtained by the fitting for different values of $n$ (number of terms of the Willams' expansion) rising from 2 to 15. The higher order terms of the series are fundamental for a better estimation of the displacement in wider areas near to the crack tip, in which the effect of the singular terms is less evident. In general, it was noted that after about 7-8 terms the series converges, and for this reason the final SIFs values were obtained by computing the average in the range between 8 th and 15 th terms.

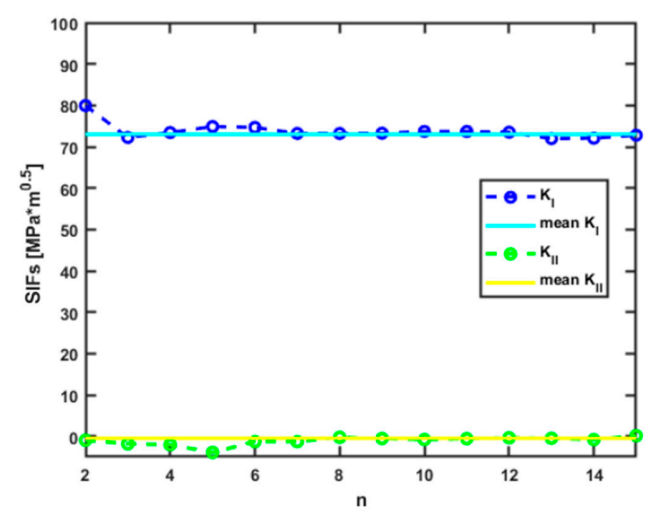

Figure 5. Stress intensity factors for values of $n$ ranging from 2 to 15 in the case of AM-0-42-42-1 specimen.

In Figure 6a comparison between the v-displacements field measured by the DIC and the one fitted by the Williams' model is shown for various combinations of Mode I and II. As expected, the $\mathrm{v}$-displacements field for Mode I (opening) is symmetric with respect to the crack axis, whereas for mixed mode I-II and Mode II (sliding) the symmetry axis rotates due the shear deformation. In the third column of Figure 6, the difference between the displacement fitted by the theoretical model and the one measured by the DIC is plotted. By ranging from Mode I to Mode II, the area where the linear elastic model overestimates the experimental displacement field grows. Figure 7 shows the u-displacement for the AM-10-42-10.2 specimen, which is more significative than the $\mathrm{v}$-displacement since Mode II is predominant. Indeed, a higher absolute error is shown for the $\mathrm{u}$-displacement compared to the $\mathrm{v}$-displacement, being higher the $\mathrm{u}$ values for Mode II with respect to the v values.

The SIFs obtained by the full displacement field (DIC) and critical fracture load (PCR) are listed in Table 3. As expected, the $K_{I C}$ measured for the specimens with the sintered crack-like notch (AM), compared to the pre-cracked one (SM), is overestimated of about $40 \%$ and $30 \%$ for the PCR and the DIC method, respectively. The reason is attributed to the larger radius of the crack-like notch compared to the one, significantly smaller, of the case of the pre-cracked specimens. However, with this specimen geometry, the pre-crack could be induced without having a deviation from its principal axis only for Mode I, and for this reason mixed mode SIFs were investigated only with crack-like notch directly induced by the AM process. For Mode I, an overestimation of about $70 \%$ of the DIC results with respect to conventional PCR method can be observed. Going from Mode I to Mode II, this discrepancy becomes negligible. 
(a)
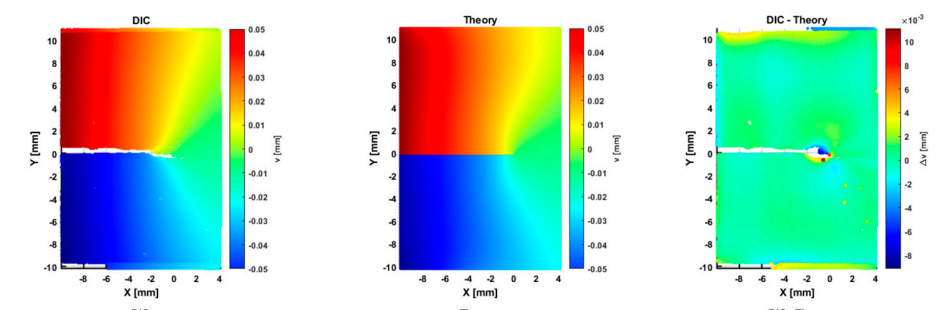

(b)
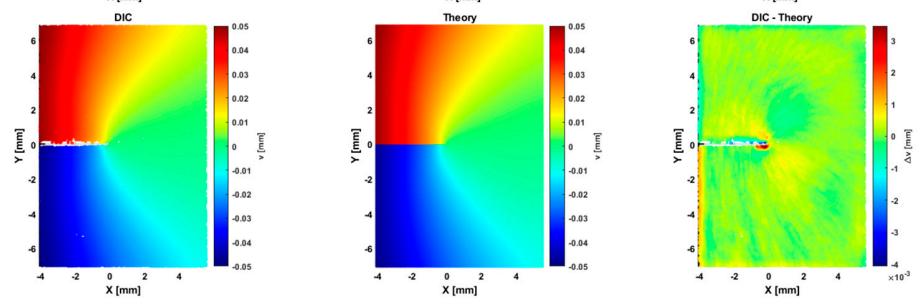

(c)
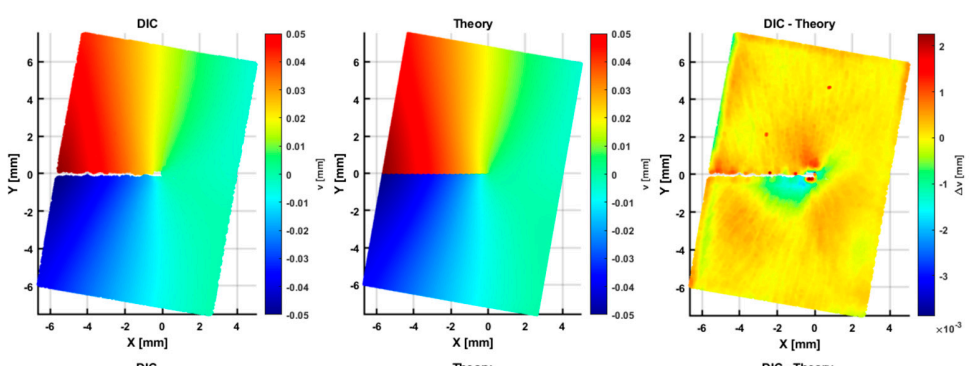

DIC

Theory
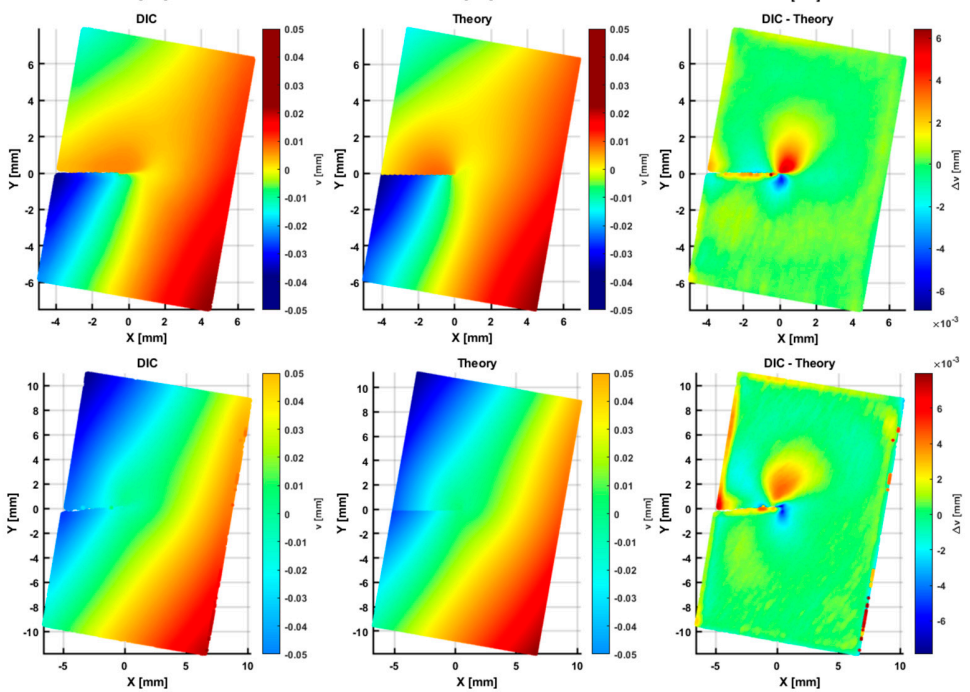

Figure 6. Comparison between the $v$-displacements field measured by the DIC and the one fitted by the Williams' model for various specimens and loading condition: (a) SM-0-42-42 (Mode I, pre-cracked); (b) AM-0-42-42 (Mode I); (c) AM-10-42-42 (mixed mode I-II); (d) AM-10-42-18 (mixed mode I-II); (e) AM-10-42-10.2 (Mode II).
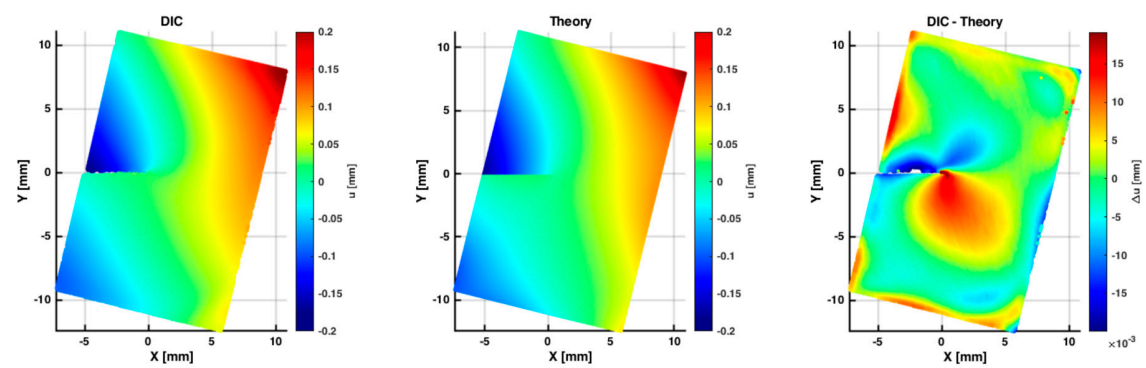

Figure 7. $u$-displacements field measured by the DIC and the one fitted by the Williams' model in the case of the specimen AM-10-42-10.2 (Mode II). 


\subsection{Generalized Mixed-Mode Local Stress Criterium}

To study the overall mixed-mode fracture toughness behavior of the sintered maraging steel, the SIFs previously obtained were plotted on the graph $K_{\mathrm{I}}-K_{\mathrm{II}}$, normalized to $K_{\mathrm{IC}}$, which is the critical SIF obtained for Mode I. As a multiaxial fracture model, the local stress criterium (LS), proposed by Yongming Liu in [27] was exploited. Classical criteria based on maximum tangential stress, as the generalized maximum tangential stress one (GMTS) [28], can only predict fracture toughness of brittle materials. On the other hand, a major advantage of the LS criterium is that it can be applied to different materials (brittle and ductile), which experience either shear or tensile dominated crack propagation. Therefore, this model can be suitable for the strong, but at the same time ductile, maraging steel. The LS mixed mode criterium is described by Equation (13), with s equal to $K_{\mathrm{IIC}} / K_{\mathrm{IC}}$. The material parameter $\mathrm{s}$ is related to the material ductility and affects the critical plane orientation. If the parameter $\mathrm{s}$ is higher than 1 , as in this case, $A=9\left(s^{2}-1\right), B=s, \Upsilon=0$. The equation 13 represents the implicit curve of the LS criterium, and the coefficient $\mathrm{s}$ can be determined numerically by fitting the experimental SIFs normalized to the $K_{\mathrm{IC}}$.

$$
\begin{gathered}
\sqrt{\left(\frac{k_{1}}{k_{I C}}\right)^{2}+\left(\frac{k_{2}}{k_{I C} S}\right)^{2}+A\left(\frac{k_{H}}{k_{I C}}\right)^{2}}=B \\
\text { where : }\left\{\begin{array}{c}
k_{1}=\frac{K_{I}}{2}(1+\cos 2 \alpha)+K_{2} \sin 2 \alpha \\
k_{2}=-\frac{K_{I}}{2} \sin 2 \alpha+K_{I I} \cos 2 \alpha ; \alpha=\beta+\gamma ; \beta=\frac{1}{2} \operatorname{arctg} \frac{2 K_{I I}}{K_{I}} \\
k^{H}=\frac{K_{I}}{3}
\end{array}\right.
\end{gathered}
$$

As can be observed in Figure 8, the LS criterium is able to fit the SIFs (of the AM specimens) obtained with both the PCR and the DIC methods.

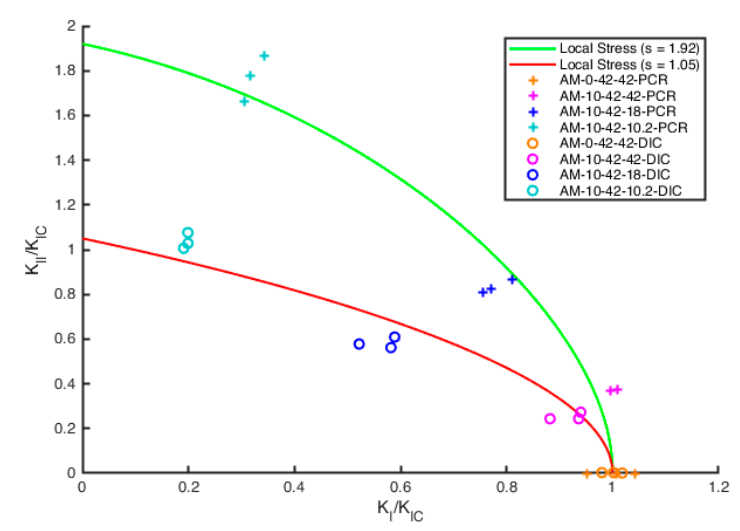

Figure 8. Fitting of the local stress criterium on the experimental data of the AM specimens.

\section{Discussion}

Pure and mixed mode I-II SIFs were evaluated for ASCB specimens of maraging steel produced by additive SLS process. SIFs of 44.7 MPa $\mathrm{m}^{0.5}$ and 79.1 MPa $\mathrm{m}^{0.5}$ were obtained with PCR methodology for Mode I and Mode II, respectively.

The feasibility of inducing crack-like notches directly during the additive manufacturing (AM) process was investigated and compared with the classical subtractive one (SM), for Mode I. The AM method could be used to induce in the specimen internal crack-like notches with any angle and shape and study three-dimensional fracture mechanics problems, which would not be feasible with conventional methods. Micrograph analysis shows that notch with a slightly sharp tip with a radius smaller than $100 \mu \mathrm{m}$ can be obtained by the AM technique. As expected, an overestimation of about $30-40 \%$ of the SIFs was measured for the crack-like notch produced by AM technique, compared to the fatigue loading pre-cracked one. Indeed, being the pre-crack sharper than the sintered one, the stress concentration at the tip is higher, and therefore the specimen would fail with a lower load, 
which corresponds to a lower SIF. Comparing the obtained results, for maraging steel, with the one reported by the author in [3], for Nylon polymer, the discrepancy between the crack-like notch induced by AM and SM technique is higher ( $30-40 \%$ vs. $2-4 \%$ ). This can be explained by the different ductility of the two studied materials. Indeed, the polymer, being more ductile, is less sensible to the sharpness of the notch.

Two different methods for evaluating the SIFs were compared: the classical one based on the critical load (PCR) and a less conventional one based on fitting of the Williams' model on the full field displacement measurement at the crack tip (DIC). This latter method could be used for non-destructive analysis by the DIC of the crack status in three dimensional structures were the stress state is difficult to be predicted. For Mode I, an overestimation of about $70 \%$ of the DIC results with respect to the conventional PCR method can be observed. While going from Mode I to Mode II this discrepancy become negligible.

It is well known in literature [29] (p. 76) that the Mode I SIF depends on the three-dimensional stress state at the crack tip. In particular, it is overestimated for thin specimens, which are in plane stress condition, whereas it decreases for increasing specimen thickness until a plateau is reached at plane strain state. Amr A. Abd-Elhady [30] conducted a three-dimensional finite element analysis on SCB specimens in order to evaluate the SIF throughout the thickness for mixed mode I/II loading condition. The Author observed that for Mode I, the geometry factor decreases moving from the mid-plane to the outer surface of the specimen, in order to compensate for the higher apparent SIF due to the plane stress condition. Conversely, for Mode II, the SIF is less dependent on the thickness and shows an opposite trend with respect to Mode I. Hence, in our case, the overestimation of the Mode I SIFs via the DIC can be attributed to the fact that the method evaluates it by exploiting the measured displacement field on the outer surface, which is on plane stress condition. This phenomenon becomes less marked varying from Mode I to Mode II according to the numerical results of Amr A. Abd-Elhady [30].

\section{Conclusions}

Mixed and Mode I and II SIFs were evaluated for maraging steel ASCB specimens produced by the additive SLS process. A non-conventional method to induce crack-like notches directly during the additive manufacturing process was proposed and validated on metal specimens. Two different methods for evaluating the SIFs were compared: the classical one based on the critical load and a less conventional one based on the fitting of the Williams' model on the full field displacement measurement at the crack tip, measured by a DIC system. Finally, the experimental results were described by the mixed mode local stress criterium.

Supplementary Materials: The MATLAB ${ }^{\circledR}$ code used for the calculation of the DIC method is online at http://www.mdpi.com/2075-4701/10/3/400/s1.

Author Contributions: Conceptualization, T.M.B. and G.M.; Funding acquisition, S.Ć.K.; Investigation, I.C. and T.M.B.; Methodology, I.C. and T.M.B.; Sample production, J.J.T., S.C..K. and N.B.; Software, I.C.; Supervision, G.M.; Visualization, N.B.; Writing—original draft, I.C. and T.M.B.; Writing—review \& editing, T.M.B. and G.M. All authors have read and agreed to the published version of the manuscript.

Funding: The research presented in this paper is part of the A_Madam Project, funded by the European Union's Horizon 2020 research and innovation program under the Marie Skłodowska-Curie action, grant agreement No. 734455.

Acknowledgments: The authors would like to thank Nenad Drvar and Josip Kos from Topomatika (Zagreb, Croatia), for the technical support on the DIC measurements. Two of the authors (N.B. and S.Ć.K.) would like to acknowledge support of the Serbian Ministry of Education, Science and Technology Development.

Conflicts of Interest: The authors declare no conflict of interest.

\section{References}

1. Kruth, J.; Mercelis, P.; van Vaerenbergh, J.; Froyen, L.; Rombouts, M. Binding mechanisms in selective laser sintering and selective laser melting. Rapid Prototyp. J. 2005, 11, 26-36. [CrossRef] 
2. Zhang, W.; Melcher, R.; Travitzky, N.; Bordia, R.K.; Greil, P. Three-dimensional printing of complex-shaped alumina/glass composites. Adv. Eng. Mater. 2009, 11, 1039-1043. [CrossRef]

3. Brugo, T.; Palazzetti, R.; Ciric-Kostic, S.; Yan, X.T.; Minak, G.; Zucchelli, A. Fracture Mechanics of laser sintered cracked polyamide for a new method to induce cracks by additive manufacturing. Polym. Test. 2016, 50, 301-308. [CrossRef]

4. Williams, J.G.; Ewing, P.D. Fracture under complex stress-The angled crack problem. Int. J. Fract. Mech. 1972, 8, 441-446. [CrossRef]

5. Papadopoulos, G.A.; Poniridis, P.I. Crack initiation under biaxial loading with higher-order approximation. Eng. Fract. Mech. 1989, 32, 351-360. [CrossRef]

6. Silva, A.L.L.; de Jesus, A.M.P.; Xavier, J.; Correia, J.A.F.O.; Fernandes, A.A. Combined analytical-numerical methodologies for the evaluation of mixed-mode (I + II) fatigue crack growth rates in structural steels. Eng. Fract. Mech. 2017, 185, 124-138. [CrossRef]

7. Aliha, M.R.M.; Ayatollahi, M.R. Brittle fracture evaluation of a fine grain cement mortar in combined tensile-shear deformation. Fatigue Fract. Eng. Mater. Struct. 2009, 32, 987-994. [CrossRef]

8. Lim, I.L.; Johnston, I.W.; Choi, S.K.; Boland, J.N. Fracture testing of a soft rock with semi-circular specimens under three-point bending. Part 2-Mixed-mode. Int. J. Rock Mech. Min. Sci. Geomech. Abstr. 1994, 31, 199-212. [CrossRef]

9. Ayatollahi, M.R.; Aliha, M.R.M.; Saghafi, H. An improved semi-circular bend specimen for investigating mixed mode brittle fracture. Eng. Fract. Mech. 2011, 78, 110-123. [CrossRef]

10. Darban, H.; Haghpanahi, M.; Assadi, A. Determination of crack tip parameters for ascb specimen under mixed mode loading using finite element method. Comput. Mater. Sci. 2011, 50, 1667-1674. [CrossRef]

11. Saghafi, H.; Monemian, S.A. New fracture toughness test covering mixed-mode conditions and positive and negative t-stresses. Int. J. Fract. 2010, 165, 135-138. [CrossRef]

12. Saghafi, H.; Zucchelli, A.; Minak, G. Evaluating fracture behavior of brittle polymeric materials using an IASCB specimen. Polym. Test. 2013, 32, 133-140. [CrossRef]

13. Moore, A.J.; Tyrer, J.R. The evaluation of fracture mechanics parameters from electronic speckle pattern interferometric fringe patterns. Opt. Lasers Eng. 1993, 19, 325-336. [CrossRef]

14. Peters, H.W.; Ranson, F.W. Digital imaging techniques in experimental stress analysis. Opt. Eng. 1982, 21, 427-431.

15. McNeill, S.R.; Peters, W.H.; Sutton, M.A. Estimation of stress intensity factor by digital image correlation. Eng. Fract. Mech. 1987, 28, 101-112. [CrossRef]

16. Muskhelishvili, N.I. Some Basic Problems of the Mathematical Theory of Elasticity, 4th ed.; Springer: Berlin, Germany, 1977.

17. Nurse, A.D.; Patterson, E.A. Determination of predominantly mode II stress intensity factors from isochromatic data. Fatigue Fract. Eng. Mater. Struct. 1993, 16, 1339-1354. [CrossRef]

18. Lopez-Crespo, P.; Shterenlikht, A.; Patterson, E.A.; Yates, J.R.; Withers, P.J. The stress intensity of mixed mode cracks determined by digital image correlation. J. Strain Anal. Eng. Des. 2008, 43, 769-780. [CrossRef]

19. Yoneyama, S.; Ogawa, T.; Kobayashi, Y. Evaluating mixed-mode stress intensity factors from full-field displacement fields obtained by optical methods. Eng. Fract. Mech. 2007, 74, 1399-1412. [CrossRef]

20. Yates, J.R.; Zanganeh, M.; Asquith, D.; Tai, Y.H. Quantifying Crack Tip Displacement Fields: T-Stress and CTOA. In Proceedings of the Crack Paths, Vicenza, Italy, 23-25 September 2009.

21. Beretta, S.; Patriarca, L.; Rabbolini, S. Stress intensity factor calculation from displacement fields. Frat. ED Integrità Strutt. 2017, 11, 269-276. [CrossRef]

22. Bonniot, T.; Doquet, V.; Mai, S.H. Determination of effective stress intensity factors under mixed-mode from digital image correlation fields in presence of contact stresses and plasticity. Strain 2020, 56, e12332. [CrossRef]

23. Croccolo, D.; de Agostinis, M.; Fini, S.; Olmi, G.; Vranic, A.; Ciric-Kostic, S. Influence of the build orientation on the fatigue strength of eos maraging steel produced by additive metal machine: How the build direction affects the fatigue strength of additive manufacturing processed parts. Fatigue Fract. Eng. Mater. Struct. 2016, 39, 637-647. [CrossRef]

24. Palanca, M.; Brugo, T.M.; Cristofolini, L. Use of digital image correlation to investigate the biomechanics of the vertebra. J. Mech. Med. Biol. 2015, 15, 1540004. [CrossRef] 
25. Williams, M.L. On the stress distribution at the base of a stationary crack. J. Appl. Mech. 1957, 24, 109-114. [CrossRef]

26. Tan, C.; Zhou, K.; Ma, W.; Zhang, P.; Liu, M.; Kuang, T. Microstructural evolution, nanoprecipitation behavior and mechanical properties of selective laser melted high-performance grade 300 maraging steel. Mater. Des. 2017, 134, 23-34. [CrossRef]

27. Liu, Y.; Mahadevan, S. Threshold stress intensity factor and crack growth rate prediction under mixed-mode loading. Eng. Fract. Mech. 2007, 74, 332-345. [CrossRef]

28. Smith, D.J.; Ayatollahi, M.R.; Pavier, M.J. The role of t-stress in brittle fracture for linear elastic materials under mixed-mode loading. Fatigue Fract. Eng. Mater. Struct. 2001, 24, 137-150. [CrossRef]

29. Anderson, T.L. Fracture Mechanics: Fundamentals and Applications, 3rd ed.; CRC Press, Taylor \& Francis Group: Boca Raton, FL, USA, 2005.

30. Abd-Elhady, A.A. Mixed mode I/II stress intensity factors through the thickness of disc type specimens. Eng. Solid Mech. 2013, 1, 119-128. [CrossRef]

(C) 2020 by the authors. Licensee MDPI, Basel, Switzerland. This article is an open access article distributed under the terms and conditions of the Creative Commons Attribution (CC BY) license (http://creativecommons.org/licenses/by/4.0/). 